BARROS \& MENEZES, v(8), no 8, p. 1581-1590, SET-DEZ, 2012.

Rev. Elet. em Gestão, Educação e Tecnologia Ambiental (e-ISSN: 2236-1170)

\title{
ANÁLISE ESTATÍSTICA DO RISCO DE MORTE POR INFECÇÃO HOSPITALAR EM GOIÂNIA
}

\author{
Victor Freitas de Azeredo Barros ${ }^{1}$, José Elmo de Menezes ${ }^{2}$ \\ ${ }^{1}$ CAPES Foundation, Ministry of Education of Brazil, Brasília/DF - Brazil; \\ PhD Student Information System and Technology, School of Engineering - University of Minho, Guimarães, \\ Portugal.vfbarros@gmail.com \\ ${ }^{2}$ Pontifícia Universidade Católica de Goiás; Instituto Federal de Educação, Ciência e Tecnologia de Goiás, campus \\ Inhumas. jelmo@ucg.br
}

http://dx.doi.org/10.5902/223611706840

Resumo - A Infecção Hospitalar é considerada um problema grave, crescendo tanto em incidência quanto em complexidade, gerando diversos tipos de implicações sociais e econômicas. O controle desta deve ser à base de atuação de todos os profissionais de saúde. A solução é um fortalecimento das Comissões de Controle de Infecção Hospitalar (CCIH). Baseado nestas problemáticas propõe-se a modelagem matemática do risco de um indivíduo ter uma Infecção Hospitalar e o risco de morte de um individuo com infeç̧ão hospitalar. Para isso, faremos um levantamento de dados via IBGE, ANVISA, entre outros, e daremos a eles um tratamento estatístico. Como ferramenta teórica, utilizaremos a Teoria de Análise de Sobrevivência, bem como os modelos estatísticos abordados nesta teoria. Como ferramenta computacional, utilizaremos o programa $\mathrm{R}$, um Software gratuito que propicia excelente ambiente para análise estatística e com recursos gráficos de alta qualidade..

Palavras chave - Infecção Hospitalar, Fatores de Risco, Modelagem Estatística, Análise de Sobrevivencia.

\begin{abstract}
The Hospital Infection is considered a serious problem, growing both in incidence as in complexity, generating many kinds of social and economic implications. The control of this must be based the all health professionals. The solution is strengthening the Commission for Hospital Infection Control (CHIC). Based on this problem is proposed mathematical modeling of the risk of a person collapse a Hospital Infection and the risk of death of a person with Hospital Infection. For this, we will do a data survey in IBGE, ANVISA, and others, and we will do with this data a statistic treatment. As theoretical tool, we will use the Theory of Survival Analysis, and too the statistical models discussed in this theory. As a computational tool, we will use the R program, free software that provides excellent means for statistics analysis and with high quality of graphical features.
\end{abstract}

Index Terms — Hospital Infection, Risk Factors, Statistical Models, Survival Analysis.

\section{INTRODUÇÃO}

A Infeç̧ão Hospitalar (IH) existe desde que surgiu os hospitais no século XIX. A elevada incidência de doenças epidêmicas que acometiam as comunidades pobres e, também, as precárias condições 
Rev. Elet. em Gestão, Educação e Tecnologia Ambiental (e-ISSN: 2236-1170)

de higiene e de saneamento em que vivia a população, foram fatores decisivos no irromper de tais infecções [1].

A Infecção Hospitalar atinge o mundo todo e representa uma das causas de morte em pacientes hospitalizados. No Brasil, segundo o Ministério da Saúde, a taxa média de infecção hospitalar é cerca de 15\%, ao passo que nos EUA e na Europa é cerca de 10\% [2]. Cabe lembrarmos, no entanto, que o índice de infecção hospitalar varia significativamente, pois está diretamente relacionada com o nível de atendimento e complexidade de cada hospital [3].

Os principais fatores que influenciam a aquisição de uma infecção são: o status imunológico; a idade (recém-nascidos e idosos são mais vulneráveis); o uso abusivo de antibióticos; os procedimentos médicos, em particular os invasivos; e as falhas nos procedimentos de controle de infecção.

Embora o controle e monitoramento dos mais variados tipos de infecções seja objeto de constante preocupação por parte do governo, bem como das agências ligadas à saúde, como, por exemplo, a Agência Nacional da Vigilância Sanitária (ANVISA) e a Organização Pan-Americana de Saúde (OPAS), e outras, ainda a muito a ser feito e estudado sobre o assunto [4].

Segundo o IBGE, 36\% das clínicas de saúde com leitos para internação no Brasil não fazem o devido controle [5]. Em Goiânia, um levantamento realizado pela Coordenação Municipal de Controle de Infecção Hospitalar nos estabelecimentos de Saúde em Goiânia (COMCIES) em 2006, detectou que apenas $20 \%$ dos estabelecimentos de saúde da Capital possuem Comissões de Controle de Infecção Hospitalar [6], contrariando assim a Portaria Federal número 2616, de 12 de maio de 1998 [7].

A realidade é que a Infecção Hospitalar representa um dos maiores problemas nos hospitais e clínicas, um surto de infecção provoca altos custos nos hospitais com uso fora do comum com antibióticos que em geral são caros, além do custo por perda da vida humana ser algo imensurável [8].

Baseado nestas problemáticas propõe-se, como objeto de pesquisa, a modelagem matemática do risco de morte de um indivíduo por Infecção Hospitalar.

Para isso, foi feito um levantamento de dados via IBGE, ANVISA, Secretaria Municipal de Saúde e outros órgãos disponíveis, e daremos a esses dados um tratamento estatístico.

Como ferramenta teórica essencial, utilizaremos a Teoria da Análise de Sobrevivência, bem como os modelos estatísticos matemáticos abordados nesta teoria, como, por exemplo, o Estimador de Kaplan-Meier, Modelos Probabilísticos, entre outros.

Como ferramenta computacional, utilizaremos o programa estatístico $\mathrm{R}$, um software gratuito e de código aberto que propicia excelente ambiente para análise estatística e com recursos gráficos de alta qualidade.

\section{MATERIAL e MÉTOdos}

Foi realizado um estudo retrospectivo, a partir do banco de dados do Hospital, computando os resultados das amostras encaminhadas para cultura, no período compreendido entre Janeiro de 2006 à Janeiro de 2008.

Foi feito um estudo descritivo que analisou a microbiota isolada em amostras de secreção traqueal, urina, sangue e outras. 
Rev. Elet. em Gestão, Educação e Tecnologia Ambiental (e-ISSN: 2236-1170)

Foram analisados 424 pacientes que apresentaram pelo menos uma amostra positiva, e a partir destes, foi feito um estudo estatístico para poder modelar matematicamente, o risco de morte por Infecção Hospitalar.

A partir destes dados foi construída uma tabela referente aos tempos de sobrevivência, em meses, de pacientes com Infecção Hospitalar e a partir desta pôde ter-se os valores estimados obtidos por meio do estimador de Kaplan-Meier da função de sobrevivência.

Com estes valores estimados obtidos por meio do estimador de Kaplan-Meier, foi construída uma segunda tabela, referente às estimativas de Kaplan-Meier das funções de sobrevivência para os dados dos pacientes com Infecção Hospitalar.

Para uma melhor visualização dos dados descritos foi construído o gráfico da função de sobrevivência estimada por Kaplan-Meier através do programa estatístico R.

Através deste gráfico foi feito um estudo com os modelos probabilísticos para ajustar os modelos apresentados (exponencial, de Weibull e log-normal) e, com base na comparação entre valores estimados e observados, decidir qual deles "melhor" explica os dados amostrais, ou seja, relacionar qual modelo que mais se aproxima à curva de sobrevivência.

Para isto, utilizamos as expressões estimadas das funções de sobrevivência para os modelos e construímos uma nova tabela mostrando as estimativas das funções de sobrevivência usando-se os modelos exponencial, de Weibull e log-normal e também o Kaplan-Meier.

Para a escolha de um dos modelos, construímos, inicialmente, os gráficos das estimativas das sobrevivências obtidas pelo método de Kaplan-Meier versus as estimativas das sobrevivências obtidas a partir dos modelos exponencial, de Weibull e log-normal.

$\mathrm{Na}$ tentativa de confirmar os resultados obtidos pelo método citado acima, construímos os gráficos linearizados e então teremos como confirmar qual modelo "melhor" representa os dados amostrais.

\section{RESUltados e DiscussõES}

A partir da coleta no banco de dados do Hospital dos pacientes que apresentaram pelo menos uma amostra positiva de Infecção Hospitalar, a partir das amostras de secreção traqueal, urina sangue e outras, de Janeiro de 2007 à Janeiro de 2008, têm-se como especificar os tempos de sobrevivência, em meses, dos 424 pacientes infectados, conforme mostrado na Tabela I.

TABELA I: TEMPOS DE SOBREVIVÊNCIA, EM MESES, DE 424 PACIENTES COM INFECÇÃO HOSPITALAR

\begin{tabular}{|c|c|c|c|c|c|c|c|c|c|c|c|c|}
\hline 1 & 1 & 1 & 2 & 2 & 2 & 2 & 2 & 2 & 2 & 2 & 2 & 3 \\
\hline 3 & 3 & 3 & 3 & 3 & 3 & 3 & 4 & 4 & 4 & 4 & 4 & 4 \\
\hline 4 & 4 & 4 & 4 & 4 & 4 & 5 & 5 & 5 & 5 & 5 & 5 & 5 \\
\hline 5 & 5 & 5 & 5 & 5 & 5 & 6 & 6 & 7 & 7 & 7 & 7 & 7 \\
\hline 8 & 8 & 8 & 8 & 8 & 8 & 8 & 8 & 8 & 8 & 9 & 9 & 9 \\
\hline 9 & 9 & 9 & 9 & 9 & 9 & 9 & 9 & 9 & 9 & 9 & 9 & 9 \\
\hline 9 & 9 & 9 & 9 & 9 & 9 & 9 & 10 & 10 & 10 & 10 & 10 & 10 \\
\hline 10 & 10 & 10 & 10 & 10 & 10 & 10 & 10 & 10 & 10 & 10 & 10 & 10 \\
\hline 10 & 10 & 11 & 11 & 11 & 11 & 11 & 11 & 11 & 11 & 11 & 11 & 11 \\
\hline 11 & 11 & 11 & 11 & 11 & 11 & 11 & 12 & 12 & 12 & 12 & 12 & 12 \\
\hline 12 & 12 & 12 & 12 & 12 & 12 & 12 & 12 & 12 & 12 & 12 & 12 & 12 \\
\hline 12 & 12 & 12 & 12 & 12 & 12 & 12 & 12 & 12 & 13 & 13 & 13 & 13 \\
\hline 13 & 13 & 13 & 13 & 13 & 13 & 13 & 13 & 13 & 13 & 13 & 13 & 13 \\
\hline 13 & 13 & 13 & 13 & 14 & 14 & 14 & 14 & 14 & 14 & 14 & 14 & 14 \\
\hline 14 & 14 & 14 & 14 & 14 & 14 & 14 & 14 & 14 & 14 & 14 & 14 & 14 \\
\hline 14 & 14 & 14 & 14 & 14 & 14 & 15 & 15 & 15 & 15 & 15 & 15 & 15 \\
\hline 15 & 15 & 15 & 15 & 15 & 15 & 15 & 15 & 15 & 15 & 15 & 15 & 15 \\
\hline
\end{tabular}


Rev. Elet. em Gestão, Educação e Tecnologia Ambiental (e-ISSN: 2236-1170)

\begin{tabular}{lllllllllllll}
\hline 15 & 15 & 15 & 15 & 15 & 15 & 15 & 15 & 15 & 15 & 15 & 15 & 15 \\
15 & 16 & 16 & 16 & 16 & 16 & 16 & 16 & 16 & 16 & 16 & 16 & 16 \\
16 & 16 & 16 & 16 & 16 & 16 & 17 & 17 & 17 & 17 & 17 & 17 & 17 \\
17 & 17 & 17 & 17 & 17 & 17 & 17 & 17 & 17 & 17 & 17 & 17 & 17 \\
17 & 17 & 17 & 17 & 17 & 18 & 18 & 18 & 18 & 18 & 18 & 18 & 18 \\
18 & 18 & 18 & 18 & 18 & 18 & 18 & 18 & 18 & 18 & 18 & 18 & 18 \\
18 & 18 & 18 & 18 & 19 & 19 & 19 & 19 & 19 & 19 & 19 & 19 & 19 \\
19 & 19 & 19 & 19 & 19 & 19 & 19 & 19 & 19 & 20 & 20 & 20 & 20 \\
20 & 20 & 20 & 20 & 20 & 20 & 20 & 20 & 20 & 20 & 20 & 20 & 20 \\
20 & 20 & 20 & 20 & 20 & 20 & 20 & 20 & 21 & 21 & 21 & 21 & 21 \\
21 & 21 & 21 & 21 & 21 & 21 & 21 & 21 & 21 & 21 & 21 & 21 & 21 \\
21 & 21 & 21 & 21 & 21 & 21 & 21 & 21 & 21 & 21 & 21 & 21 & 21 \\
21 & 22 & 22 & 22 & 22 & 22 & 22 & 22 & 22 & 22 & 22 & 22 & 22 \\
22 & 22 & 22 & 22 & 22 & 22 & 22 & 22 & 22 & 22 & 22 & 22 & 22 \\
22 & 22 & 23 & 23 & 23 & 23 & 23 & 23 & 23 & 23 & 23 & 23 & 23 \\
24 & 24 & 24 & 24 & 24 & 24 & 24 & 25 & 25 & 25 & - & - & - \\
\hline
\end{tabular}

A partir destes dados, utilizamos o estimador de Kaplan-Meier estimado por,

$$
\hat{S}_{K M}(t)=\prod_{r, t_{r}<t} \frac{n_{i}-d_{i}}{n_{i}}
$$

onde $n_{i}$ representa o número de indivíduos vivos até o tempo $t_{i}, d_{i}$ representa o número de mortes no tempo $t_{i}$ e $t_{r}$ representa o maior tempo de sobrevivência, para obtermos os tempos de sobrevivência, bem como o desvio-padrão e os limites de confiança, conforme mostra a Tabela II.

TABELA II: ESTIMATIVAS DE KAPLAN-MEIER DAS FUNÇÕES DE SOBREVIVÊNCIA PARA OS DADOS DA TABELA I

\begin{tabular}{llllllll}
\hline $\mathrm{t}$ (meses) & $\begin{array}{l}\text { Número } \\
\text { Pacientes }\end{array}$ & $\begin{array}{l}\text { Número } \\
\text { Mortes }\end{array}$ & Sobrev. & $\begin{array}{l}\text { Desvio } \\
\text { Padrão }\end{array}$ & Limite Inferior & $\begin{array}{l}\text { Limite } \\
\text { Superior }\end{array}$ \\
\hline 01 & {$[01,02)$} & 424 & 3 & 0.99292 & 0.00407 & 0.98498 & 1.0000 \\
02 & {$[02,03)$} & 421 & 9 & 0.97170 & 0.00805 & 0.95604 & 0.9876 \\
03 & {$[03,04)$} & 412 & 8 & 0.95283 & 0.01030 & 0.93286 & 0.9732 \\
04 & {$[04,05)$} & 404 & 12 & 0.92453 & 0.01283 & 0.89972 & 0.9500 \\
05 & {$[05,06)$} & 392 & 13 & 0.89387 & 0.01496 & 0.86503 & 0.9237 \\
06 & {$[06,07)$} & 379 & 2 & 0.88915 & 0.01525 & 0.85976 & 0.9195 \\
07 & {$[07,08)$} & 377 & 5 & 0.87736 & 0.01593 & 0.84668 & 0.9091 \\
08 & {$[08,09)$} & 372 & 10 & 0.85377 & 0.01716 & 0.82080 & 0.8881 \\
09 & {$[09,10)$} & 362 & 23 & 0.79953 & 0.01944 & 0.76231 & 0.8386 \\
10 & {$[10,11)$} & 339 & 21 & 0.75000 & 0.02103 & 0.70990 & 0.7924 \\
11 & {$[11,12)$} & 318 & 18 & 0.70755 & 0.02209 & 0.66555 & 0.7522 \\
12 & {$[12,13)$} & 300 & 26 & 0.64623 & 0.02322 & 0.60228 & 0.6934 \\
13 & {$[13,14)$} & 274 & 21 & 0.59670 & 0.02382 & 0.55178 & 0.6453 \\
14 & {$[14,15)$} & 253 & 28 & 0.53066 & 0.02424 & 0.48522 & 0.5804 \\
15 & {$[15,16)$} & 225 & 34 & 0.45047 & 0.02416 & 0.40552 & 0.5004 \\
16 & {$[16,17)$} & 191 & 18 & 0.40802 & 0.02387 & 0.36382 & 0.4576 \\
17 & {$[17,18)$} & 173 & 25 & 0.34906 & 0.02315 & 0.30651 & 0.3975 \\
18 & {$[18,19)$} & 148 & 25 & 0.29009 & 0.02204 & 0.24996 & 0.3367 \\
19 & {$[19,20)$} & 123 & 18 & 0.24764 & 0.02096 & 0.20978 & 0.2923 \\
20 & {$[20,21)$} & 105 & 25 & 0.18868 & 0.01900 & 0.15488 & 0.2298 \\
\hline
\end{tabular}


Rev. Elet. em Gestão, Educação e Tecnologia Ambiental (e-ISSN: 2236-1170)

\begin{tabular}{llllllll}
\hline 21 & {$[21,22)$} & 80 & 32 & 0.11321 & 0.01539 & 0.08673 & 0.1478 \\
22 & {$[22,23)$} & 48 & 27 & 0.04953 & 0.01054 & 0.03264 & 0.0752 \\
23 & {$[23,24)$} & 21 & 11 & 0.02358 & 0.00737 & 0.01278 & 0.0435 \\
24 & {$[24,25)$} & 10 & 7 & 0.00708 & 0.00407 & 0.00229 & 0.0219 \\
25 & {$[25,26)$} & 3 & 3 & 0.00000 & - & - & - \\
\hline
\end{tabular}

O comportamento do tempo de sobrevivência pode estar associado a várias causas físicas e pode ser de difícil representação matemática. Neste contexto, temos várias distribuições de probabilidade que são usualmente utilizadas para modelar de forma acessível dados de sobrevivência, que são os modelos exponencial, de Weibull e log-normal [9]. Estas expressões das estimativas das funções de sobrevivência para os modelos exponencial, de Weibull e log-normal, são respectivamente,

$$
\begin{aligned}
& \hat{S}(t)_{e}=\exp \{-t / 14,51\}, \\
& \hat{S}(t)_{w}=\exp \left\{-(t / 16,24)^{2,74}\right\}, \\
& \hat{S}(t)_{\ln }=\Phi\left[\frac{-(\log (t)-2,55)}{0,58}\right],
\end{aligned}
$$

Em [8] usa-se um exemplo de aplicação de um conjunto de técnicas estatísticas para dados da área da saúde, mas especificamente, calculam-se os tempos de reincidência de um grupo de pacientes com câncer de bexiga submetido a procedimentos cirúrgicos feito a laser. Aqui usaremos a mesma seqüência cronológica, estimando a curva de sobrevivência pelo modelo de Kaplan-Meier e para a linearização usaremos os modelos exponencial, de Weibull e log-normal.

A Figura 1 mostra o gráfico da função de sobrevivência estimada por meio de Kaplan-Meier, a partir dos tempos de sobrevivência.

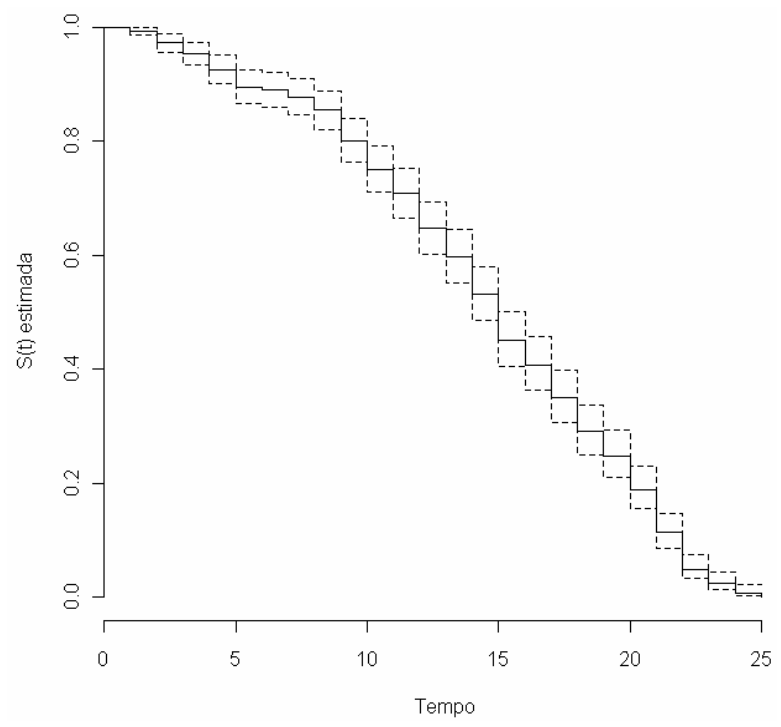

FIGURA 1> GRÁFICO DA ESTIMATIVA DE KAPLAN-MEIER 
Rev. Elet. em Gestão, Educação e Tecnologia Ambiental (e-ISSN: 2236-1170)

Assim como os tempos de sobrevivência de Kaplan-Meier, calculamos também os tempos de sobrevivência para os modelos exponencial, de Weibull e log-normal a partir das expressões das estimativas das funções de sobrevivência para os modelos, como podemos ver na Tabela III.

TABELA III. ESTIMATIVAS DA SOBREVIVÊNCIA USANDO-SE O ESTIMADOR DE KAPLAN-MEIER E OS MODELOS EXPONENCIAL, DE WEIBULL E LOG-NORMAL

\begin{tabular}{|c|c|c|c|c|c|}
\hline \multicolumn{2}{|c|}{$\mathrm{t}$ (meses) } & Kaplan-Meier & Exponencial & Weibull & Log-normal \\
\hline 01 & {$[01,02)$} & 0.992924528 & 0.9334216 & 0.99951974 & $5.726168 \mathrm{e}^{-06}$ \\
\hline 02 & {$[02,03)$} & 0.971698113 & 0.8712759 & 0.99679373 & $1.200606 \mathrm{e}^{-08}$ \\
\hline 03 & {$[03,04)$} & 0.952830189 & 0.8132678 & 0.99028949 & $1.716204 e^{-10}$ \\
\hline 04 & {$[04,05)$} & 0.924528302 & 0.7591217 & 0.97875975 & $6.315064 \mathrm{e}^{-12}$ \\
\hline 05 & {$[05,06)$} & 0.893867925 & 0.7085806 & 0.96119609 & $4.131459 \mathrm{e}^{-13}$ \\
\hline 06 & {$[06,07)$} & 0.889150943 & 0.6614045 & 0.93684807 & $3.997655 e^{-14}$ \\
\hline 07 & {$[07,08)$} & 0.877358491 & 0.6173692 & 0.90525852 & $5.145584 e^{-15}$ \\
\hline 08 & {$[08,09)$} & 0.853773585 & 0.5762658 & 0.86629869 & $8.238481 e^{-16}$ \\
\hline 09 & {$[09,10)$} & 0.799528302 & 0.5378990 & 0.82019332 & $1.567974 e^{-16}$ \\
\hline 10 & {$[10,11)$} & 0.75000000 & 0.5020865 & 0.76752818 & $3.434945 \mathrm{e}^{-17}$ \\
\hline 11 & {$[11,12)$} & 0.707547170 & 0.4686584 & 0.70923480 & $8.458092 e^{-18}$ \\
\hline 12 & {$[12,13)$} & 0.646226415 & 0.4374559 & 0.64654985 & $2.299084 \mathrm{e}^{-18}$ \\
\hline 13 & {$[13,14)$} & 0.596698113 & 0.4083308 & 0.58094936 & $6.802317 e^{-19}$ \\
\hline 14 & {$[14,15)$} & 0.530660377 & 0.3811448 & 0.51406172 & $2.166305 \mathrm{e}^{-19}$ \\
\hline 15 & {$[15,16)$} & 0.450471698 & 0.3557688 & 0.44756588 & $7.358858 e^{-20}$ \\
\hline 16 & {$[16,17)$} & 0.408018868 & 0.3320823 & 0.38308425 & $2.646667 \mathrm{e}^{-20}$ \\
\hline 17 & {$[17,18)$} & 0.349056604 & 0.3099728 & 0.32208056 & $1.001609 \mathrm{e}^{-20}$ \\
\hline 18 & {$[18,19)$} & 0.290094340 & 0.2893353 & 0.26577288 & $3.967758 e^{-21}$ \\
\hline 19 & {$[19,20)$} & 0.247641509 & 0.2700718 & 0.21507071 & $1.638012 \mathrm{e}^{-21}$ \\
\hline 20 & {$[20,21)$} & 0.188679245 & 0.2520909 & 0.17054139 & $7.020448 e^{-22}$ \\
\hline 21 & {$[21,22)$} & 0.113207547 & 0.2353071 & 0.13240808 & $3.113598 e^{-22}$ \\
\hline 22 & {$[22,23)$} & 0.049528302 & 0.2196407 & 0.10057682 & $1.424849 \mathrm{e}^{-22}$ \\
\hline 23 & {$[23,24)$} & 0.023584906 & 0.2050174 & 0.07468731 & $6.711129 \mathrm{e}^{-23}$ \\
\hline 24 & {$[24,25)$} & 0.007075472 & 0.1913677 & 0.05417912 & $3.246263 e^{-23}$ \\
\hline 25 & {$[25,26)$} & 0.00000000 & 0.1786267 & 0.03836432 & $1.609470 \mathrm{e}^{-23}$ \\
\hline
\end{tabular}

Para a escolha de um dos modelos, construímos os gráficos das estimativas de sobrevivência obtidas pelo método de Kaplan-Meier versus as estimativas de sobrevivência obtidas a partir dos modelos exponencial, de Weibull e log-normal, conforme a Figura 2. 
Rev. Elet. em Gestão, Educação e Tecnologia Ambiental (e-ISSN: 2236-1170)
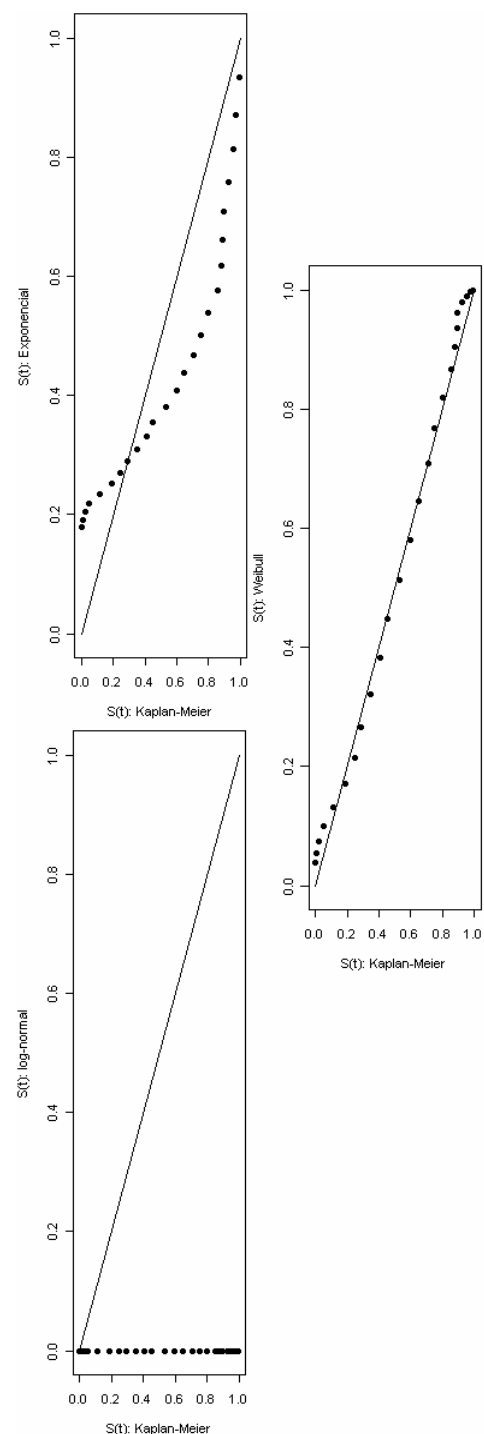

FIGURA 2.GRÁFICOS DAS SOBREVIVÊNCIAS ESTIMADAS POR KAPLAN-MEIER VERSUS AS SOBREVIVÊNCIAS ESTIMADAS PELOS MODELOS EXPONENCIAL DE WEIBULL E LOG-NORMAL

A partir deste gráfico é possível observar que o modelo log-normal parece não ser adequado para esses dados, pois a curva se apresenta um tanto afastada da reta $y=x$. Por outro lado, os modelos exponencial e de Weibull acompanham mais de perto a reta, indicando ser, possivelmente, um dos modelos mais adequado para os dados sob estudo.

Os dois modelos, exponencial e de Weibull, apresentaram resultados similares. Para que assim confirme qual apresentará ajustes satisfatórios, faz-se um novo gráfico com as curvas de sobrevivência estimadas por meio do ajuste de ambos os modelos versus a curva de sobrevivência estimada pelo modelo de Kaplan-Meier que podem ser observadas na Figura 3 e Figura 4. 
Rev. Elet. em Gestão, Educação e Tecnologia Ambiental (e-ISSN: 2236-1170)

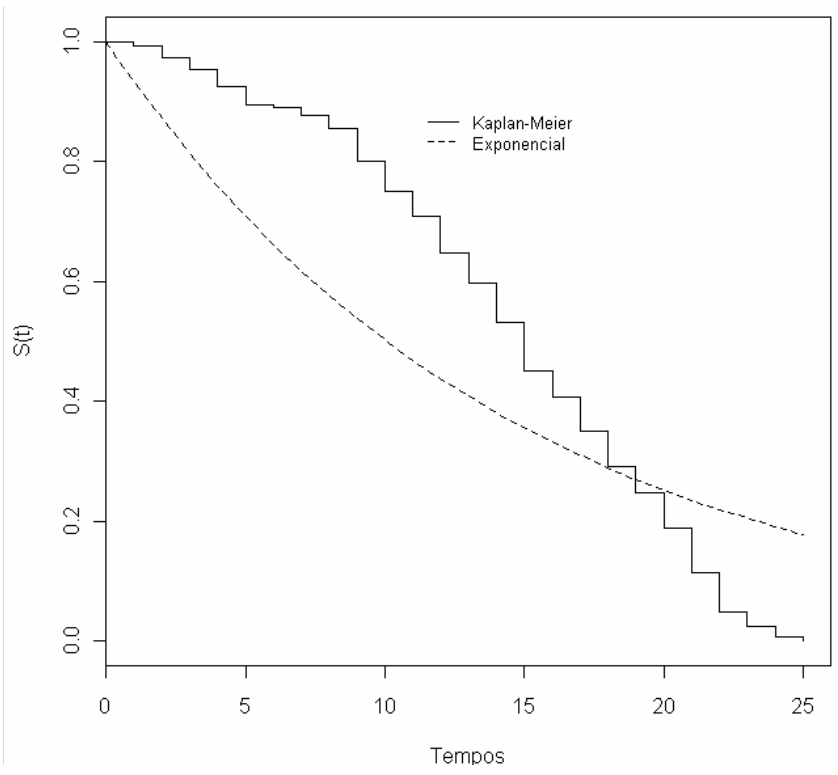

FIGURA 3.GRÁFICO DA CURVA DE SOBREVIVÊNCIA ESTIMADA PELO MODELO EXPONENCIAL VERSUS A CURVA DE SOBREVIVÊNCIA DE KAPLAN-MEIER

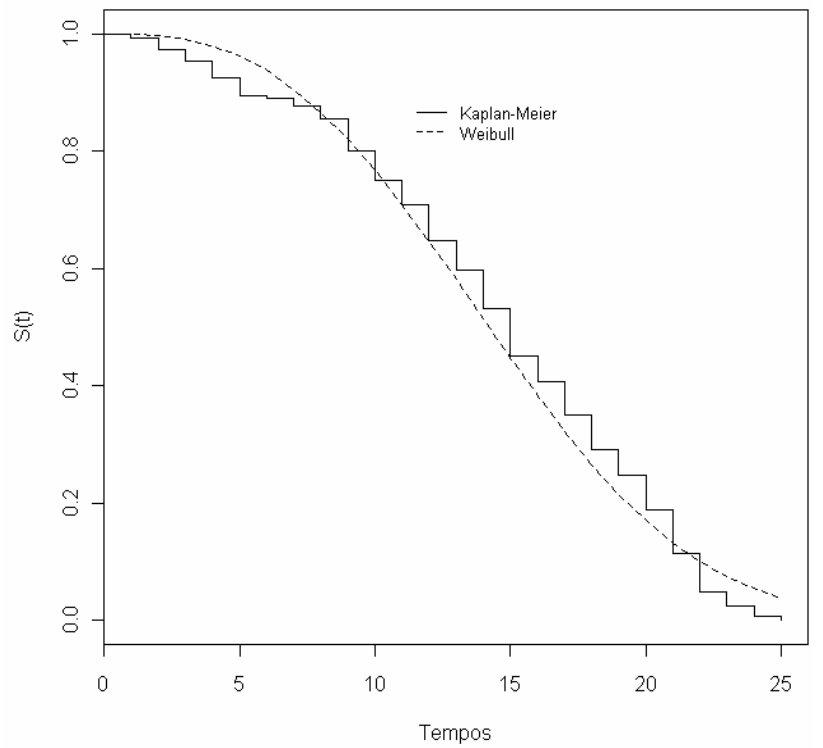

FIGURA 4. GRÁFICO DA CURVA DE SOBREVIVÊNCIA ESTIMADA PELO MODELO DE WEIBULL VERSUS A CURVA DE SOBREVIVÊNCIA DE KAPLAN-MEIER

Por fim, podemos confirmar que o modelo de Weibull é o mais adequado, pois, é o modelo cujos pontos da função de sobrevivência estão mais próximos dos valores obtidos pelo estimador de Kaplan-Meier.

Encontram-se resultados parecidos ao deste estudo em [8], ao analisar o tempo de reincidência de um grupo de pacientes com câncer de bexiga que foram submetidos a um 


\section{ating}

BARROS \& MENEZES, v(8), no 8, p. 1581-1590, SET-DEZ, 2012.

Rev. Elet. em Gestão, Educação e Tecnologia Ambiental (e-ISSN: 2236-1170)

procedimento cirúrgico feito por laser, porém, os modelos que apresentaram os melhores ajustes foram os modelos de Weibull e log-normal.

\section{CONCLUSÃO}

Com a utilização do estimador de Kaplan-Meier foi possível analisar e calcular os tempos de sobrevivência deste paciente, verificando que a curva estimada é do tipo escada, portanto, mostra as probabilidades de sobrevivência constantes entre os tempos de mortes adjacentes, mas crescentes a cada tempo distinto, portanto, esta é válida para modelar estatisticamente os tempos de vida de sistemas.

Verificou-se também que o programa $\mathrm{R}$ é confiável para o desenvolvimento dos modelos probabilísticos e do estimador de Kaplan-Meier, e de acordo com o estimador e modelos propostos conclui-se que o modelo Weibull é o que mais se ajusta a função de sobrevivência tendo-se o valor de 2,74 como parâmetro de forma, gama, e o valor de 16,24 como parâmetros de escala, alpha.

Com isso podemos observar, por exemplo, que uma estimativa para $\mathrm{S}(12)$ usando-se o modelo de Weibull é 64,7\%. Esta mesma estimativa usando-se o estimador de Kaplan-Meier é de 64,6\%. Estes valores são bastante próximos e significa que um paciente tem uma probabilidade de morrer de $64,65 \%$ após 12 meses em que o paciente contraiu a infecção.

Atualmente, a análise de sobrevivência é cada vez mais desejável, devido ao número de aplicações de análise de sobrevivência em medicina. Sendo assim, no trabalho aqui analisado foi possível obter uma realização de estudos de análise de sobrevivência, e através da modelagem desses sistemas, verificar as taxas de risco e tempos de sobrevivência dos pacientes com Infecção Hospitalar.

\section{REFERÊNCIAS}

[1] Moura, M.E.B., Ramos, M.N., Sousa, C.M.M, Silva, A.O., Alves, M.S.C.F., “Infecção Hospitalar no Olhar de Enfermeiros Portugueses: Representações Sociais", Texto Contexto Enferm, Florianópolis, Out-Dez, 17(4), 743-9, 2008.

[2] Levi, C.E., "Manual de Microbiologia Clínica para o Controle de Infecção em Serviços de Saúde", IX Congresso Brasileiro de Controle de Infecção e Epidemiologia Hospitalar, Salvador, 30 de Agosto a 3 de Setembro de 2004.

[3] Martins, P., "Epidemiologia das Infecções Hospitalares em Centro de Terapia Intensiva de Adulto", Universidade Federal de Minas Gerais, Dissertação de Mestrado, 2006.

[4] Santos, A.A.M., "O Modelo Brasileiro para o Controle das Infeç̧ões Hospitalares: Após Vinte Anos de Legislação, onde estamos e para onde vamos?", Universidade Federal de Minas Gerais, Dissertação de Mestrado, 2006.

[5] Araújo, E.S., "Estatísticas da Saúde: Assistência Médico-Sanitária”, Departamento de População e Indicadores Sociais, Rio de Janeiro: IBGE, 162 p, 2006. 
[6] Arantes, A., Carvalho, E.S., Medeiros, E.A.S., Farhat, C.K., Mantese, O.C., "O Uso de Diagramas de Controle na Vigilância Epidemiológica das Infecções Hospitalares", Revista Saúde Pública, 37(6): 768-74, 2003.

[7] Ministério da Saúde (BR), "Expede na Forma de Anexos Diretriz e Normas para a Prevenção e Controle das Infeç̧ões Hospitalares: Portaria no 2.616, de 12 de maio de 1998", Diário Oficial da União, 1998.

[8] Banderó Filho, V.C., Reschke, C.R., Höner, R., “Perfil Epidemiológico das Infecções Hospitalares na Unidade de Terapia Intensiva Infantil do Hospital de Caridade e Beneficência de Cachoeira do Sul”, RS, Brasil, RBAC, vol. 38(4): 267-270, 2006.

[9] Colossimo, E.A., Giolo, S.R., “Análise de Sobrevivência Aplicada”, 1ạ ed., São Paulo, SP: Blücher, 370p, 2006. 